\title{
Characterization of an optical fiber bundle sensor
}

\author{
Laurence Bergougnoux, Jacqueline Misguich-Ripault, and Jean-Luc Firpo ${ }^{\text {a) }}$ \\ Groupe d'Instrumentation des Procédés et Systèmes en Ecoulement, Institut Universitaire des Systèmes \\ Thermiques Industriels, UMR 6595, Technopôle de Château Gombert, 13453 Marseille, Cedex 13, France
}

(Received 4 November 1997; accepted for publication 4 February 1998)

\begin{abstract}
An optical fiber sensor made of a central emitting fiber and two concentric crowns of receiving fibers is presented. This sensor is mainly used to measure high solid volume concentrations in suspension. The aim of this article is to accurately characterize the geometrical properties of this sensor comparing experimental data and simulation. Experimentally, the sensor is used as a displacement sensor with a metallic mirror as a target. This geometrical problem is simulated by a Monte Carlo method, which takes into account: (1) The experimental determination of the angular distribution of light intensity for the emitting fiber; (2) The finite size of fibers by means of a numerical integration procedure; and (3) The multiple reflections of light on the fiber sections with an explicit use of Fresnel's factors. By fitting the experimental data with the results of the simulation, we can determine the geometrical properties of the probe and photon efficiency (voltage output versus number of launched photons) of the device. Finally, we discuss the use of this sensor as a displacement sensor. In this case a simple electronic device is given to gain a single valued response of the probe when the distance between the target and the sensor is varied. In this framework, the nature of the scattering target is also discussed. (C) 1998 American Institute of Physics. [S0034-6748(98)00705-9]
\end{abstract}

\section{INTRODUCTION}

In a previous article, ${ }^{1}$ we have presented an optical fiber sensor devoted to concentration measurement in concentrated suspensions. This sensor is made of an optical fiber bundle: the central fiber brings the light and two concentric fiber crowns collect the light backscattered by particles in suspension. Such a sensor can also be used as a displacement sensor. In this case, the concentric crowns collect the light re-emitted by a target and the sensor response depends on the target distance. Frank ${ }^{2}$ and Kissinger ${ }^{3}$ originally found the principle of this displacement sensor. The sensors differ from one another in the geometrical arrangement of fibers. Actually, two main classes of sensors exist:

(a) The emitting and receiving fibers are randomly distributed in the bundle. See for instance Shimamoto and Tanaka ${ }^{4}$ and Bailly-Salins. ${ }^{5}$

(b) The fibers in the bundle are distributed with one central emitting fiber and a concentric crown of receiving fibers $\left(\right.$ Cook and $\left.\mathrm{Ham}^{6}\right)$.

Recently, Anghel $^{7}$ et al. have presented a device with one fiber devoted to the emission and two fibers at different distances from the emitting fiber which gather the light reemitted by the target.

In this article, the behavior of our sensor as a displacement one is analyzed at experimental and theoretical levels. In the case of concentration measurements, the theoretical determination of the sensor response is ruled by the multiple scattering of light by the particles in suspension. ${ }^{1}$ In the displacement case, the problem is easily handled, so the focus is

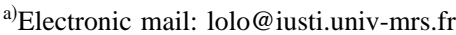

put on the characterization of the sensor itself. In Sec. II, we describe the experimental device. In Sec. III, the Monte Carlo calculation based on photon launching allows the light flux gathered by a fiber to be calculated, set at a distance $x$ of the emitting fiber, when the target is placed at a distance $d$ from the sensor. This response is given in terms of the number of photons gathered by the receiving fibers (i.e., in an arbitrary unit of light flux). In Sec. IV, the experimental results are compared with Monte Carlo calculations. Finally, the performances of this displacement sensor are discussed.

\section{EXPERIMENTAL DEVICE}

\section{A. The sensor}

Our sensor is made of the same plastic optical fibers (FORETEC PG-U-FB750) whose features $(R f, \beta)$ are summarized in Table I. The bundle is formed by a central emitting fiber surrounded by two concentric crowns devoted to reception, so that the total number of fibers equals 19 (see Fig. 1 and Fig. 2).

For displacement measurement, the light from a Hewlett-Packard HLMP-8103 LED of $650 \mathrm{~nm}$ peak wavelength and $7^{\circ}$ emitting angle is coupled onto the emitting fiber. This light-emitting-diode is supplied at $F=0.9 \mathrm{kHz}$ frequency with $8 \mathrm{~V} \mathrm{dc}$ potential, i.e., $40 \mathrm{~mA}$ forward current $I_{0}$ imposed by a resistance $R$. Consequently, $4.2 \mu \mathrm{W}$ are sent onto the fiber.

TABLE I. Characteristics of optical fibers.

\begin{tabular}{cccc}
\hline $\begin{array}{c}\text { Core radius } R f \\
(\mu \mathrm{m})\end{array}$ & $\begin{array}{c}\text { Clad thickness } \\
(\mu \mathrm{m})\end{array}$ & $\begin{array}{c}\text { Numerical aperture } \\
\sin \beta \text { in air }(-)\end{array}$ & $\begin{array}{c}\text { Attenuation at } \\
640 \mathrm{~nm}(\mathrm{~dB} / \mathrm{km})\end{array}$ \\
\hline 375 & 5 & 0.47 & 160 \\
\hline \hline
\end{tabular}




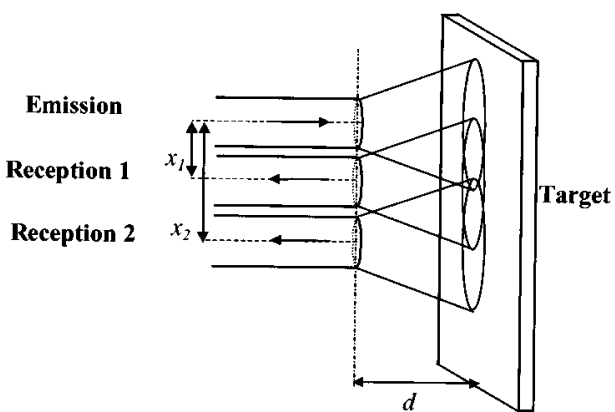

FIG. 1. Bundle optical fiber displacement sensor principle.

The two crowns of receiving fibers are connected to two Hamamatsu S1406-06 photodiode/amplifier detectors with a $0.38 \mathrm{~A} / \mathrm{W}$ radiant sensitivity at $650 \mathrm{~nm}$. The photodiode short circuit current is converted into a dc potential. Under these conditions, the average gain $g_{1}$ of the detectors is equal to $0.6 \mathrm{~V} / \mu \mathrm{W}$.

The effect of the ambient light is canceled by the use of a synchronous detection device.

\section{B. Experimental determination of light distribution in the acceptance cone $\beta$}

For the Monte Carlo process, we have to know the angular distribution of light intensity in the acceptance cone of the emitting fiber. Some previous calculations have shown that, when an isotropic distribution in the acceptance cone $\beta$ at emission is assumed, we cannot fit the experimental curves. At this stage, to obtain the most accurate determination of the sensor response, we must experimentally determine the angular distribution $P_{\Delta \epsilon, \Delta \phi}(\epsilon)$ of light emitted by the emitting fiber in a solid angle $\Delta \epsilon, \Delta \phi$ around the $\epsilon$ direction. This measurement is performed for different angles $\epsilon$ in the range of $(0-0.52 \mathrm{rad})$ between the emitting fiber and a photodetector. Figure 3 shows the experimental data $(\boldsymbol{\square})$ for $P_{s}(\epsilon) \sin (\epsilon) / P_{s}(\epsilon=0)$ (i.e., proportional to the probability density that a photon is launched in a direction $\theta$ in the cone of aperture $\epsilon, \epsilon+\Delta \epsilon$ ) and the fit curve (-) given by the polynomial relationship:

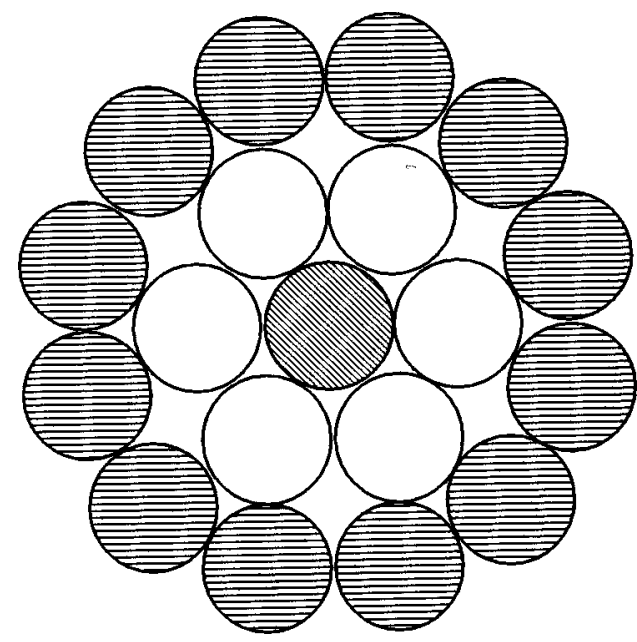

FIG. 2. Optical fiber bundle configuration with the emitting fiber and the $O$ two crowns of receiving fibers

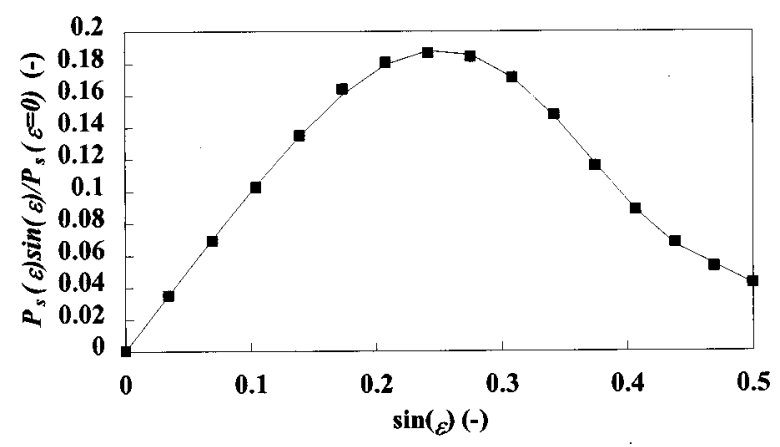

FIG. 3. Angular distribution of light at the end of the emitting fiber: experimental data and (-) polynomial fit curve $f(\epsilon)$.

$$
\begin{aligned}
f(\epsilon)= & 1.018 \epsilon-1.685 \epsilon^{3}-59.885 \epsilon^{5}+337.545 \epsilon^{7} \\
& -518.687 \epsilon^{9} .
\end{aligned}
$$

\section{Crown distance determination}

Our sensor is a home made device. Some imperfections occur mainly in the distance between the crown fibers and the central fiber. In order to evaluate these distances accurately, the sensor head was examined microscopically. Thus the mean distances between the central fiber and the crowns are, respectively,

$$
x_{1}=800 \pm 10 \mu \mathrm{m}, \quad x_{2}=1590 \pm 20 \mu \mathrm{m} .
$$

\section{Measurement procedure}

The displacement measurements are carried out on a Micro Controle optical bench with a precision translation stage equal to $20 \mathrm{~mm}$ travel and $10 \mu \mathrm{m}$ positioning resolution (see Fig. 4). The target is either an aluminized plane mirror assumed to be perfectly reflective or a scattering plane made of a paper sheet stuck on a metallic plane named paper screen.

The voltage $V_{S}$ is measured in a $(0-15 \mathrm{~mm})$ displacement range for each fiber crown.

\section{MONTE CARLO CALCULATION}

The theoretical determination of light flux gathered by receiving fibers versus the distance between target and sensor involves calculating complicated multiple integrals. For instance, in Anghel $^{7}$ et al., a calculation is performed when the fiber emits isotropically. Indeed, we must take into account

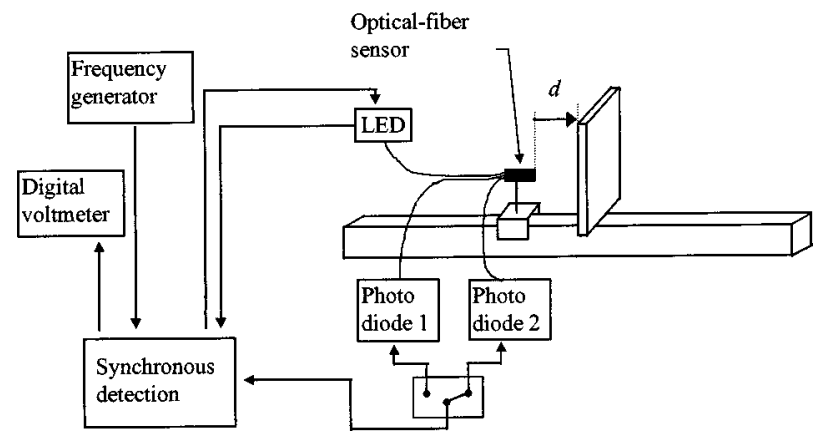

FIG. 4. Schematic diagram of the displacement measuring system. 


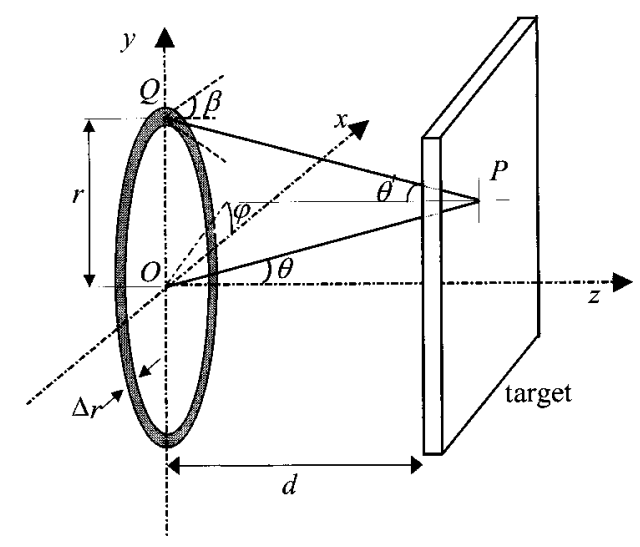

FIG. 5. $O$ is the emitting point of photons. The scattering plane is placed at a distance $d$ from the $z=0$ plane. $O P Q$ is the photon path. $P$ belongs to the scattering plane; $Q$ is in the receiving plane at a distance $r$ from $O .(\theta, \varphi)$ are the angles of the emitting direction in spherical coordinates.

the finite fiber-section size, the angular distribution of emitted light intensity, and the scattering nature of the target. Monte Carlo methods are a good means of evaluating these kinds of integrals, and give a physical understanding of this calculation. In this framework, the problem is considered in terms of photon launching. The photon path is analogous to the pencil of radiation of the classical radiative transfer. ${ }^{8}$

Following the idea initiated by Groenhius et al., ${ }^{9}$ a version of this model capable of describing the multiple light scattering in concentrated suspensions has been previously given (Bergougnoux et al. ${ }^{1}$ ). Here, a version adapted to the case of a displacement sensor is presented in order to calibrate our sensor.

\section{A. Model description}

We calculate the radial distribution of photons in the reception $(z=0)$ plane due to the scattering by a parallel plane placed at a distance $z=d$ from the pin point source in air $(n=1)$ (see Fig. 5). L. Bergougnoux's Ph.D. thesis ${ }^{10}$ gives a description of a simplified version (isotropic distribution of light source, with no multiple reflections on the fiber sections) of this model. In the following, $\rho_{i}$ represents a random variable belonging to a sequence of pseudo random numbers uniformly distributed on $[0,1]$.

The underlying hypotheses are:

\section{The source}

First, we consider a point source located in $O$. The numerical integration procedure described in Sec. III C 2, allows the simulation results to be extended to finite size fibers. This point emits photons in direction $(\theta, \varphi)$ according to the experimental determination of the angular distribution of light emitted by the central fiber. Von Neumann's rejection method $^{11}$ is used to choose the direction $\theta$ of the photon. The use of this method requires the knowledge of a function proportional to the probability density such as the polynomial fit (1). $\varphi$ is chosen uniformly distributed on $[-\pi, \pi]$ in order to maintain the axial symmetry,

$$
\varphi=2 \pi\left(\rho_{1}-0.5\right) \text {. }
$$

\section{The target}

A plane parallel to the fiber section idealizes the target. This plane re-emits the photon in two different ways:

(a) According to the optical geometrical reflection law. In this case we simply have in our coordinate system:

$$
\begin{aligned}
& \theta^{\prime}=\pi-\theta, \\
& \varphi^{\prime}=\varphi .
\end{aligned}
$$

This case is named case 1 . The metallic mirror is well described by these assumptions.

(b) The Lambert hypothesis (i.e., constant Luminance). This means that the scattered photon leaves the target in a direction characterized by the angles $\left(\theta^{\prime}, \varphi^{\prime}\right)$ given by

$$
\begin{aligned}
& \theta^{\prime}=\pi-\frac{1}{2} \arccos \left(1-2 \rho_{2}\right), \\
& \varphi^{\prime}=2 \pi\left(\rho_{3}-0.5\right) .
\end{aligned}
$$

In the following, this case will be referenced as case 2 . These relations, as it will be shown, are not sufficient to describe the paper screen well.

\section{The receiving plane}

A photon received in the $z=0$ plane is counted, if its direction is within the acceptance cone $\beta$ of the receiving fiber. In case 1 every photon is obviously accepted, whereas in case 2, some photons are rejected because the Lambertian plane radiates in any direction. So, without any light absorption by the target, with the Lambertian screen, the gathered intensity is lower.

It is also necessary to introduce the possibility of multiple reflections on the fiber sections. Without any light reflection on the fiber sections, the second crown of receiving fibers should not collect light up to a distance $d_{\min }=R f / \operatorname{tg} \beta$ assuming that the central fiber and a fiber of the second crown are separated by approximately $2 R f$ (i.e., the core diameter of the fiber). But a tiny signal is observed between 0 and $d_{\min }$ for the second crown. In the simulation, we have considered that the reception plane is partially reflecting with Fresnel's factors: ${ }^{12}$

$$
\begin{aligned}
& r_{1}=\frac{\cos \alpha-m \cos \alpha^{\prime}}{\cos \alpha+m \cos \alpha^{\prime}}, \\
& r_{2}=\frac{m \cos \alpha-\cos \alpha^{\prime}}{m \cos \alpha+\cos \alpha^{\prime}} .
\end{aligned}
$$

So for the light intensity of a non polarized beam:

$$
R=\frac{1}{2}\left(r_{1}^{2}+r_{2}^{2}\right) .
$$

In these expressions $\alpha$ and $\alpha^{\prime}$ are, respectively, the incident and bending angles of ray and $m=1.492$ the refractive index of core fibers relative to air. With our notations, $\alpha=\theta-\pi$. Our Monte Carlo procedure takes into account this possibility: the photon is re-emitted by the reception plane with a probability $R$ according to the optical geometrical law of reflection. In the case $1, R$ is about 0.04 for all possible directions of light between $[-\beta,+\beta]$. 


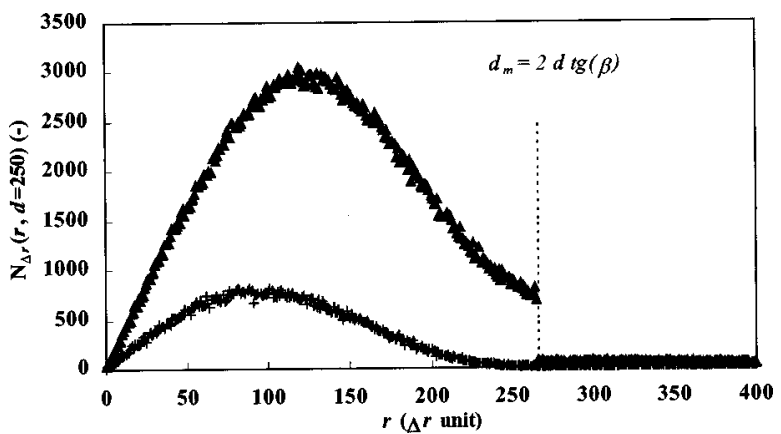

FIG. 6. Photon radial distribution $N_{\Delta r}(r, d)$ for a reflecting plane $(\mathbf{\Delta})$ and a Lambertian plane $(+)$ put at the same distance $d=250$ of the sensor with reflections on the fiber section. Total number of launched photons is $N_{\mathrm{ph}}$ $=500000$.

\section{B. Results}

This model is clearly symmetrical with respect to the $z$ axis. So, the number of photons which fall in a ring of radius $r$ and thickness $\Delta r$ is determined, and we note $N_{\Delta r}(r, d)$ this number. The results of the Monte Carlo calculation are presented in Fig. 6 with a total number of launched photons equal to 500000 , respectively, in case $1(\mathbf{\Delta})$ and $2(+)$ and multiple reflections. In this figure, the $x$ axis stands for the distance $r$ between the emitting point $O$ and the receiving point $Q$ in $\Delta r$ unit; whereas the $y$ axis is the photon distribution $N_{\Delta r}(r, d)$ obtained with $d=250$ ( $\Delta r$ unit), the distance between the plane $z=0$ and the target is the same.

These results show the influence of the target type on the distribution $N_{\Delta r}(r, d)$.

\section{Exact relations}

In a Monte Carlo calculation, it is useful to find some exact relations that allow the calculation time to be minimized and the accuracy of the method to be maximized. It is the purpose of the two subsequent paragraphs.

\section{Similarity law}

Generally, light scattering by a homogeneous plane is only dependent on incident and emergent angles. This is true for case 1 and case 2 . So, when a homothetic transformation (center $O$ and ratio $k$ ) is performed, the scattering plane is placed at distance $k d$ from the source and the photon is received at a distance $k r$ in the plane. Thus, all the photons received between $r$ and $r+\Delta r$, now fall between $k r$ and $k r+k \Delta r$. So for an integer $k$ we have

$$
N_{\Delta r}\left(r, \frac{d}{k}\right)=\sum_{i=0}^{k-1} N_{\Delta r}(k r+i \Delta r, d) .
$$

If we choose the different radii $r$ as successive multiples of $\Delta r$, with Eq. (9) we can simply deduce the radial distribution of the photons for distance $d / k$ from the distribution gained for the distance $d$. Thus, the Monte Carlo calculation will be performed once and once only, with a large distance $d$ with respect to the actual distances between the sensor and the target.

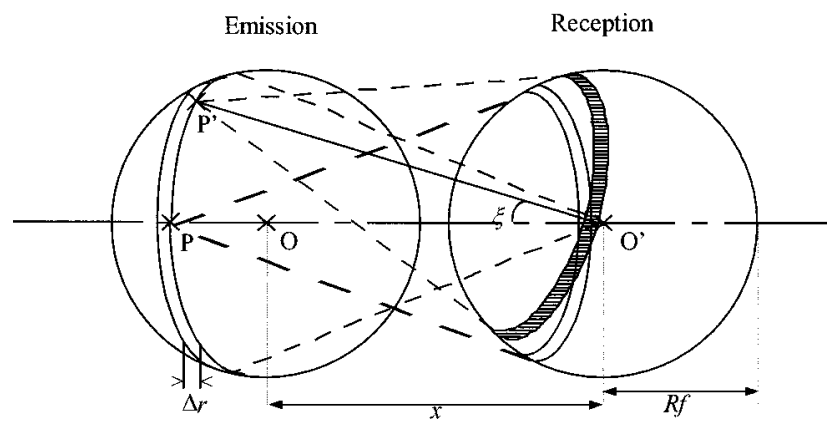

FIG. 7. Schematic diagram of the numerical integration on the emitting and receiving fiber sections.

\section{Numerical integration over the fibers}

As mentioned above, this procedure takes into account the finite emitting and receiving fiber sizes. Figure 7 shows the principle of numerical integration over two fiber sections with the same radius $R f$ and $x$ apart. For point $P$ of the emitting fiber, photons arriving at distance $r$ in a slice of thickness $\Delta r$ on the receiving fiber are counted. Thus, $r$ is varied to describe all the receiving fiber section. For the numerical integration on the emitting fiber, a simple observation is made. Point $P^{\prime}$ of the emission, obtained by a rotation of center $O^{\prime}$ and angle $\alpha$, gives the same number of photons with $(r, \Delta r)$ as with point $P$. So we can consider that every part of size $\Delta r \times \Delta r$ of the emitting fiber launches $N_{\text {ph }}$ photons.

\section{COMPARISON BETWEEN THE MEASUREMENTS AND SIMULATION}

In this section, the experimental curves of the output signal $V_{S}$ versus distance $d$ for each crown are compared with Monte Carlo process calculations. At this stage, the sensor characterization will be carried out with the metallic mirror. The latter is a better approximation of an ideal reflecting plane than the paper screen of a pure Lambertian one (see Sec. IV A). All simulation files, used later on, have been obtained from 1000000 photons gathered by the receiving fibers.

\section{A. Results}

With the parameter choice given in Secs. II A and II C, for the mirror target we obtain the results summarized in Fig. 8 . For each crown, we simply choose the best proportionality coefficient between the voltage output and the number of photons gathered by a fiber placed, respectively, at distance $x_{1}=800 \mu \mathrm{m}$ and $x_{2}=1600 \mu \mathrm{m}$.

In Table II we show this proportionality coefficient, named efficiency factor, for the two crowns and two targets. Because the area of the second crown is twice the area of the first crown, we would expect the efficiency factor for the second crown to be the double of the first crown coefficient. In fact, we have a discrepancy due to the linkage between the fiber and photodiode, which is different for each channel.

In the experiments, the uncertainty about the choice of origin $(d=0)$ implies a lack of accuracy when the measurements are duplicated. This effect may be estimated at $\pm 50 \mu \mathrm{m}$ over distances and $\pm 25 \mathrm{mV}$ over voltage output. 


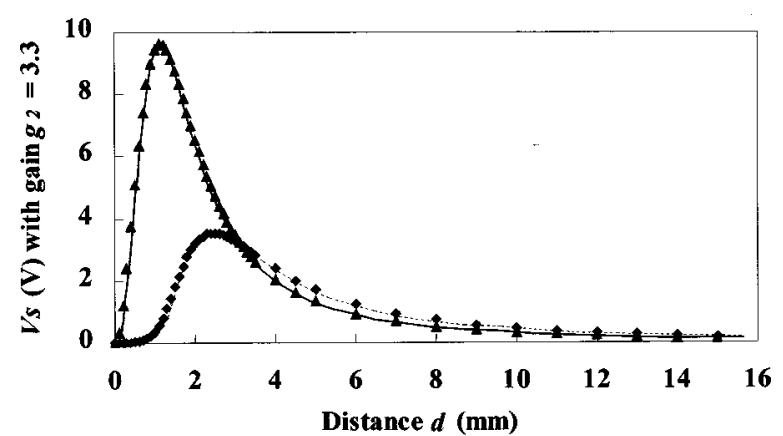

FIG. 8. Case 1: Mirror-Comparison between $(\boldsymbol{\Lambda}, \boldsymbol{\bullet})$ experimental results and $(-, \cdots)$ numerical simulation as a function of target distance $d$. Numerical results are obtained: (-) with $x_{1}=800 \mu \mathrm{m}$ and $(\cdots)$ with $x_{2}$ $=1600 \mu \mathrm{m}$.

We have to take into account this fact to compare experimental with simulation results. Thus, for the two crowns, the comparisons agree perfectly, since theoretical predictions are always within the error margin mentioned above. Particularly, we note that the surface state of the fiber section, neglected in the simulation, is not a relevant parameter. In fact, on one hand, the light scattered by a surface defect of emitting fiber is lost when it is emitted outside the aperture cone; on the other hand, the reflected light is lost when it reaches an irregularity of the receiving fibers. At the first order, this modifies only the proportionality coefficient between the model calculation and experimental results (i.e., the sensor efficiency). Nevertheless, for the second crown, when the multiple reflections are dominant $(d<1 \mathrm{~mm})$, the surface imperfections could possibly explain the discrepancy between the calculation results and the experimental data.

For the paper screen, the results are also in good agreement with the experimental data (see Fig. 9), if this target is assumed to be not purely a Lambertian screen. According to the data presented in Desvignes, ${ }^{13}$ it is assumed that the screen behaves like a mixed screen with a probability 0.88 for the Lambertian one and 0.12 for the perfectly reflecting one. The sensor response depends on the nature of the target as shown in Table III.

\section{B. Displacement sensor}

When this sensor is used for displacement measurements, the output of the first crown or second one can be used. But, it is better to work with the logarithm of the ratio of the two photodiode output voltages. In so doing, we have a single valued response of the output versus the distance. Moreover, the disturbances due to source fluctuations and, to some extent, the influence of ambient light are directly eliminated. So, the electronic device is simpler and the sensor becomes easily portable.

Figure 10 shows the output given by a log amplifier

TABLE II. Efficiency factor in volts by photon.

\begin{tabular}{ccc}
\hline \multicolumn{1}{c}{ Target } & First crown & Second crown \\
\hline Metallic mirror & $2.95 \times 10^{-8}$ & $4.14 \times 10^{-8}$ \\
Paper screen & $2.92 \times 10^{-8}$ & $4.19 \times 10^{-8}$ \\
\hline
\end{tabular}

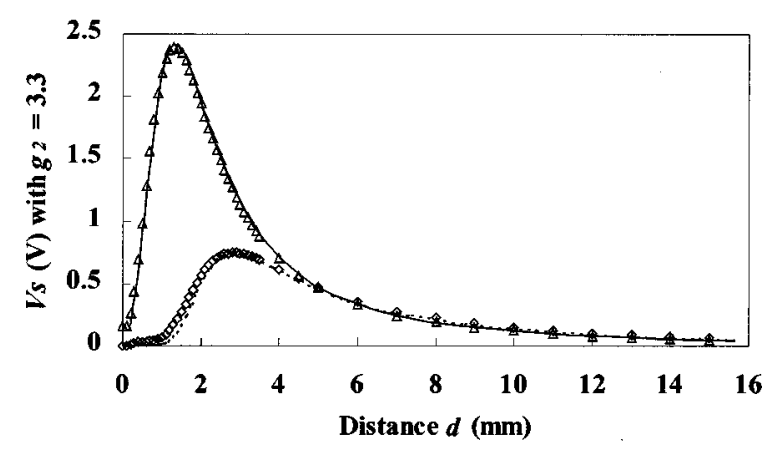

FIG. 9. Case 2: Lambertian target-Comparison between $(\triangle, \diamond)$ experimental results and $(-, \cdots)$ numerical simulation as a function of target distance $d$. Numerical results are the same: (-) with $x_{1}=800 \mu \mathrm{m}$; and $(\cdots)$ with $x_{2}=1600 \mu \mathrm{m}$.

Burr-Brown LOG100 for both screens. In the range $(0.8-4$ $\mathrm{mm})$ the sensor has a good sensitivity named $\Delta V / \Delta d$. This is evaluated by fitting with a third degree polynomial relationship:

For the mirror:

$$
\begin{gathered}
V_{\mathrm{Mir}}=-19.276+14.346 d-3.595 d^{2}+0.314 d^{3} \\
d \in(0.8 \mathrm{~mm}, 4 \mathrm{~mm}) .
\end{gathered}
$$

For the paper screen:

$$
\begin{gathered}
V_{\text {Pap }}=-15.073+10.282 d-2.411 d^{2}+0.198 d^{3} \\
d \in(0.8 \mathrm{~mm}, 4 \mathrm{~mm}) .
\end{gathered}
$$

So $\Delta V / \Delta d$ is at $d=2 \mathrm{~mm}$, respectively, equal to $3.73 \mathrm{~V} / \mathrm{mm}$ and $2.22 \mathrm{~V} / \mathrm{mm}$. Consequently, a displacement of $5 \mu \mathrm{m}$ could be easily appreciated $(\Delta V \approx 10 \mathrm{mV})$.

Because of the influence of the nature of the target, it is necessary to calibrate the device with the same target as the one used in the measurements. A mirror is better than another target type because light intensity is conserved.

\section{DISCUSSION}

In this article, we present a simple displacement sensor and the means to calculate the sensor response directly.

As mentioned in the introduction, our sensor is mainly devoted to the measurement of a solid fraction in concentrated transparent flows. For instance, this sensor is used by the Cemagref for measuring the solid transport by torrential swelling. ${ }^{14}$ In this case, the sensor is calibrated in the laboratory. But, for concentration measurements in a circulating fluidized bed, because of the turbulent nature of the flow, it is impossible to get a homogeneous distribution of particles. So, it is very difficult ${ }^{15}$ to obtain a calibration curve (i.e., voltage output versus concentration) experimentally. Taking

TABLE III. Paper screen and metallic mirror.

\begin{tabular}{lccccc}
\hline \hline \multirow{2}{*}{ Target } & \multicolumn{2}{c}{ Maximum abscissa (mm) } & & \multicolumn{2}{c}{ Maximum amplitude (V) } \\
\cline { 2 - 3 } \cline { 5 - 6 } & First crown & Second crown & & First crown & Second crown \\
\hline Metallic mirror & 1.15 & 2.45 & & 9.62 & 3.53 \\
Paper screen & 1.35 & 2.85 & & 2.40 & 0.75 \\
\hline \hline
\end{tabular}




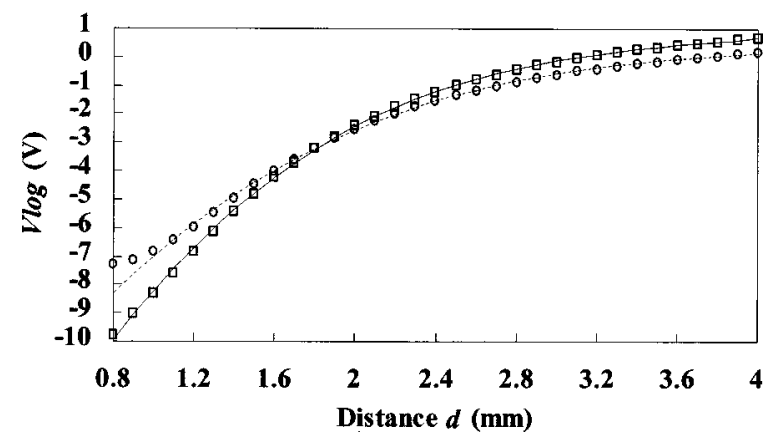

FIG. 10. Experimental results obtained with the logarithmic amplifier vs distance $d$ for the both targets: $(\square)$ mirror and $(\bigcirc)$ Lambertian screen. Fit curves given by (-) $V_{\text {Mir }}$ and $(\cdots) V_{\text {Pap }}$.

into account the good results of the Monte Carlo calculations, we can reasonably consider determining it by an $a b$ initio calculation. In Bergougnoux et al. ${ }^{1}$ we dealt with silica particles and the absorption rate was an adjustable parameter for nonspherical particles. For spherical glass particles generally used in a circulating fluidized bed, Mie's theory is fully valid, and no adjustable parameter is required. The better the characterization of the sensor, the better the results of simulation will be. The main purpose of this article is to achieve this characterization.

\section{ACKNOWLEDGMENTS}

This research was supported by the Cemagref and the Conseil Régional Provence Alpes Côte d'Azur. The authors are with the Université de Provence, Institut Universitaire des Systèmes Thermiques Industriels, Unité Mixte de Recherche 6595, Groupe d'Instrumentation des Systèmes en Ecoulements Turbidimétrie, Technopôle de ChâteauGombert, 5 rue Enrico Fermi, 13453 Marseille, France.

${ }^{1}$ L. Bergougnoux, J. Misguich-Ripault, J. L. Firpo, and J. André, Appl. Opt. 35, 1735 (1996).

${ }^{2}$ W. E. Frank, U.S. Patent No. 3,273,447 (20 September 1966).

${ }^{3}$ C. D. Kissinger, U.S. Patent No. 3,327,584 (27 June 1967).

${ }^{4}$ A. Shimamoto and K. Tanaka, Appl. Opt. 35, 6767 (1996).

${ }^{5}$ R. Bailly-Salins, Rev. Sci. Instrum. 46, 879 (1975).

${ }^{6}$ R. O. Cook and C. W. Hamm, Appl. Opt. 18, 3230 (1979).

${ }^{7}$ F. Anghel, D. Pavelescu, K. T. V. Grattan, and A. W. Palmer, Rev. Sci. Instrum. 68, 3583 (1997).

${ }^{8}$ S. Chandrasekhar, Radiative Transfer (Dover, New York, 1960), pp. 912.

${ }^{9}$ R. A. J. Groenhuis, H. A. Fewerda, and J. J. Ten Bosch, Appl. Opt. 22, 2456 (1983)

${ }^{10}$ L. Bergougnoux, Ph.D. dissertation, Université de Provence, Marseille, 1995 (unpublished).

${ }^{11}$ I. Lux and L. Koblinger, Monte Carlo Particles Transport Methods: Neutron and Photon Calculations (CRC, Boca Raton, 1991), pp. 9-12.

${ }^{12}$ H. C. Van de Hulst, Light Scattering by Small Particles (Wiley, New York, 1957), pp. 203-204.

${ }^{13}$ F. Desvignes, Rayonnements optiques Radiométrie Photométrie (Masson, Paris, 1991), pp. 90, 91.

${ }^{14}$ L. Bergougnoux, J. Misguich-Ripault, J.-L. Firpo, and J. André, in Réseau Erosion Bull. 17, edited by E. Roose, G. de Noni, and J. M. Lamachere (Orston, Montpellier, 1997).

${ }^{15}$ J. Hong and Y. Tomita, Powder Technol. 83, 85 (1995). 